\title{
DIFFEOMORPHISM OF AFFINE CONNECTED SPACES WHICH PRESERVED RIEMANNIAN AND RICCI CURVATURE TENSORS
}

\author{
V. E. BEREZOVSKI, S. BÁCSÓ, AND J. MIKEŠ
}

Received 27 April, 2016

\begin{abstract}
In this paper we study the preserving of Riemannian and Ricci tensors with respect to a diffeomorphism of spaces with affine connection. We consider geodesic and almost geodesic mappings of the first type. The basic equations of these maps form a closed system of Cauchy type in covariant derivatives. We determine the quantity of essential (substantial) parameters on which the general solution of this problem depends.
\end{abstract}

2010 Mathematics Subject Classification: 53B20; 53B21; 53B30; 53C26

Keywords: preserving, Riemannian tensor, Ricci tensor, manifold with affine connection

\section{INTRODUCTION}

The theory of geodesic mappings was developed in the works of T. Thomas, H. Weyl, L.P. Eisenhart, P.A. Shirokov, K. Yano, A.Z. Petrov, A.S. Solodovnikov, N.S. Sinyukov, A.V. Aminova, J. Mikeš, I.G. Shandra, S.E. Stepanov and others, see [1012, 14-16, 18-20,22, 24].

The problems raised in the study of geodesic maps were developed by V.F. Kagan, V. Vranceanu, P.K. Rashevsky, L.Ya. Shapiro, V.D. Vedenyapin, A.Z. Petrov and others. In particular, the concept of quasi-geodesic mapping was introduced by A.Z. Petrov. Close to it there are holomorphically projective mappings of Kähler spaces, considered originally by T. Otsuki and Y. Tashiro. See [15, 17, 19, 20,23].

It natural generalization of these classes there are almost geodesic mappings introduced by N.S. Sinyukov [20]. Recently investigated almost geodesic mappings V.S. Shadny, V.S. Sobchuk, N.V. Yablonska, J. Mikeš, V.E. Berezovski, M.S. Stankovic, L.S. Velimirovic, M.L. Zlatanovic [1-9, 19,21].

\section{BASIC CONCEPTS OF THE THEORY OF DIFFEOMORPHISM OF AFFINE CONNECTED SPACES AND THEIR TYPES}

We consider an $n$-dimensional torsion-free affine connected space in the coordinate system $(x)$, and assume that $n \geq 2$, further on all functions are sufficiently smooth.

The paper was supported by the project IGA PrF 2014016 Palacký University Olomouc. 
We can find the basic concepts of the theory of geodesic and of almost geodesic mappings in $[15,19,20]$.

Suppose, that an affine connected space $A_{n}$ admits a diffeomorphism $f$ onto an affine connected space $\bar{A}_{n}$ in a common coordinate system $x=\left(x^{1}, \ldots, x^{n}\right)$.

Let us

$$
P_{i j}^{h}(x)=\bar{\Gamma}_{i j}^{h}(x)-\Gamma_{i j}^{h}(x)
$$

given, where $\bar{\Gamma}_{i j}^{h}(x)$ and $\Gamma_{i j}^{h}(x)$ are components of objects of connections of affine connected spaces $A_{n}$ and $\bar{A}_{n}$ respectively in common coordinate system $(x), P_{i j}^{h}$ is a the deformation tensor.

Definition 1. A curve, defined in an $n$-dimensional affine connected space $A_{n}$, is called geodesic, if its tangent vector is parallel along it.

Definition 2. A curve, defined in an affine connected space $A_{n}(n>2)$, is called almost geodesic, if exists along it a two-dimensional parallel plane containing its tangent vector.

Definition 3. Diffeomorphism $f$ between two manifolds $A_{n}$ and $\bar{A}_{n}$ with affine connections is called geodesic mapping if any geodesic curve of $A_{n}$ is mapped onto a geodesic curve of $\bar{A}_{n}$ and vice versa.

Diffeomorphism $f$ is geodesic mapping if and only if the deformation tensor $P_{i j}^{h}$ in a common coordinate system has the following forms

$$
P_{i j}^{h}(x)=\psi_{i}(x) \delta_{j}^{h}+\psi_{j}(x) \delta_{i}^{h},
$$

where $\delta_{i}^{h}$ is Kronecker symbol and $\psi_{i}$ is a covariant vector field.

If $\psi_{i} \equiv 0$, then $f$ is an affine mapping.

Definition 4. Diffeomorphism $f: A_{n} \rightarrow \bar{A}_{n} \quad(n>2)$ is called almost geodesic mapping, if any geodesic curve of $A_{n}$ is mapped onto an almost geodesic curve of $\bar{A}_{n}$.

Diffeomorphism $f: A_{n} \rightarrow \bar{A}_{n}$ is almost geodesic if and only if the deformation tensor $P_{i j}^{h}$ in a common coordinate system has the following forms

$$
A_{\alpha \beta \gamma}^{h} \lambda^{\alpha} \lambda^{\beta} \lambda^{\gamma}=a P_{\alpha \beta}^{h} \lambda^{\alpha} \lambda^{\beta}+b \lambda^{h},
$$

where

$$
A_{i j k}^{h}=P_{i j, k}^{h}+P_{i j}^{\alpha} P_{\alpha k}^{h},
$$

$\bar{\Gamma}_{i j}^{h}(x)$ and $\Gamma_{i j}^{h}(x)$ are affine connections of spaces $A_{n}$ and $\bar{A}_{n}, \lambda^{h}$ is an arbitrary vector, $a$ and $b$ are some functions dependent on $x^{h}$ and $\lambda^{h}$.

Here and after the symbol ", " means covariant derivation in $A_{n}$.

N.S. Sinyukov [20] defined three types of almost geodesic mappings $\pi_{1}, \pi_{2}, \pi_{3}$ from the basic equations. It is well known $[3,6,8]$, if $n>5$ there exist only these three types. 
Almost geodesic mapping of type $\pi_{1}$ is characterized by the conditions

$$
A_{(i j k)}^{h}=a_{(i j} \delta_{k)}^{h}+b_{(i} P_{j k)}^{h},
$$

where $a_{i j}$ is some symmetric tensor, $b_{i}$ is some covector, the bracket $(i j k)$ denotes the symmetrization of the indices $i, j, k$ (without division).

If in (2.5) the covector $b_{i}$ is equal to zero, then the mapping is called cannonical. It is well known, that arbitrary almost geodesic mapping of type $\pi_{1}$ can be represented in the form of a composition of a canonical almost geodesic mapping of type $\pi_{1}$ and a geodesic mapping [20].

\section{CONDITION OF REPRESENTATION OF RIEMANNIAN AND RICCI TENSORS} WITH RESPECT TO THE DIFFEOMORPHISM OF THE AFFINE CONNECTED SPACE

From equations (2.1) we obtain a relation between Riemannian tensors $R_{i j k}^{h}$ and $\bar{R}_{i j k}^{h}$ of spaces $A_{n}$ and $\bar{A}_{n}$ respectively:

$$
\bar{R}_{i j k}^{h}=R_{i j k}^{h}+P_{i[k, j]}^{h}+P_{\alpha[j}^{h} P_{k] i}^{\alpha},
$$

where $[j k]$ denotes alternation of the indices $j, k$ (without division).

Then the relation (3.1) can be written as

$$
\bar{R}_{i j k}^{h}=R_{i j k}^{h}-A_{i[j k]}^{h} .
$$

Therefore, we have

Theorem 1. The Riemannian tensor is preserved under diffeomorphism $f: A_{n} \rightarrow$ $\bar{A}_{n}$ if and only if

$$
A_{i j k}^{h}=A_{i k j}^{h} .
$$

Contracting by indices $h$ and $k$ we obtain

$$
\bar{R}_{i j k}=R_{i j}-A_{i[j k]}^{h} .
$$

where $R_{i j}$ and $\bar{R}_{i j}$ are Ricci tensors of spaces $A_{n}$ and $\bar{A}_{n}$ respectively.

So we get

Theorem 2. The Ricci tensor is preserved under diffeomorphism $f: A_{n} \rightarrow \bar{A}_{n}$ if and only if tensor $A_{i j k}^{h}$ satisfies the following conditions

$$
A_{i j \alpha}^{\alpha}=A_{i \alpha j}^{\alpha}
$$




\section{PRESERVATION OF THE RiEMANN AND RiCCI TENSORS With RESPECT TO THE GEODESIC MAPPINGS OF THE AFFINE CONNECTED SPACE}

Consider the geodesic mappings which are characterized by the equations (2.2). Form such mappings tensors of the form

$$
A_{i j k}^{h}=\delta_{i}^{h} \psi_{j, k}+\delta_{j}^{h} \psi_{i, k}+2 \delta_{k}^{h} \psi_{i} \psi_{j}+\delta_{i}^{h} \psi_{j} \psi_{k}+\delta_{j}^{h} \psi_{i} \psi_{k} .
$$

On the basis of Theorem 1 Riemannian tensor $R_{i j k}^{h}$ is invariant with respect to geodesic mapping, if condition (3.3) satisfies.

Substituting (4.1) into (3.3) we have

$$
\delta_{j}^{h}\left(\psi_{i, k}-\psi_{i} \psi_{k}\right)+\delta_{i}^{h}\left(\psi_{j, k}-\psi_{j} \psi_{k}\right)-\delta_{i}^{h}\left(\psi_{k, j}-\psi_{k} \psi_{j}\right)-\delta_{k}^{h}\left(\psi_{i, j}-\psi_{i} \psi_{j}\right)=0 .
$$

After contraction (4.2) by indices $h$ and $i$ we obtain

$$
\psi_{j, k}-\psi_{k, j}=0 \text {. }
$$

Transvecting (4.2) by indices $h$ and $j$, we get

$$
n\left(\psi_{i, k}-\psi_{i} \psi_{k}\right)-\left(\psi_{i, k}-\psi_{k} \psi_{i}\right)=0 .
$$

From (4.3) and (4.4) follows

Therefore

$$
\psi_{i, k}=\psi_{i} \psi_{k}
$$

Theorem 3. An affine connected space $A_{n}$ admits geodesic mapping into an another affine connected space $\bar{A}_{n}$ preserving Riemmanian tensor if and only if the Cauchy type system (4.5) has a solution with respect to functions $\psi_{i}(x)$.

On the basis of Theorem 2, the Ricci tensor $R_{i j}$ of an affine connected space $A_{n}$ is invariant with respect to geodesic mappings if satisfies condition (3.5).

Substituting (4.1) into (3.5) we get

$$
\psi_{i, j}-\psi_{j} \psi_{i}=n\left(\psi_{i, j}-\psi_{i} \psi_{j}\right)
$$

From (4.5) and (4.6) we obtain (4.4).

The above leads to

Theorem 4. An affine connected space $A_{n}$ admits geodesic mapping into an another affine connected space $\bar{A}_{n}$ preserving the Ricci tensor if and only if Cauchy type system (4.4) has a solution with respect to functions $\psi_{i}(x)$.

Integrability conditions of equations (4.4) are the following

$$
\psi_{a} R_{i j k}^{h}=0 \text {. }
$$

The general solution of Cauchy type system (4.4) depends on no more than $n$ substantial parameters.

From (2.3) and (2.4) follows 
Theorem 5. If under a geodesic mapping of an affine connected space $A_{n}$ into an affine connected space $\bar{A}_{n}$ Ricci tensor is an invariant geometric object, then Riemannian tensor also is an invariant geometric object.

This result can be achieved, if we consider the Weyl tensor.

$$
W_{i j k}^{h}=R_{i j k}^{h}+\frac{1}{n+1} \delta_{i}^{h} R_{[j k]}-\frac{1}{n^{2}-1}\left[\left(n R_{i j}+R_{j i}\right) \delta_{k}^{h}-\left(n R_{i k}+R_{k i}\right) \delta_{j}^{h}\right],
$$

which is an invariant object under geodesic mappings.

Space $A_{n}$ which admits geodesic mapping preserving Riemannian and Ricci tensors, is characterized by the following interesting property.

Theorem 6. In an affine connected space which admits geodesic mappings preserving Riemannian and Ricci tensors, there exists a covariant constant vector field.

Indeed, from (4.3) we have that covector $\psi_{i}$ is locally gradient, that is, there exists function $\Psi(x)$ such that $\psi_{i}(x)=\partial_{i} \Psi(x)$ where $\partial_{i}=\partial / \partial x^{i}$.

We introduce function $\Phi(x)=e^{-\Psi(x)}$ and we can see on the basis of condition (4.5) satisfies $\Phi_{i, j}=0$. Thus covector field $\varphi_{i}=\partial_{i} \Phi(x)$ is a covariant constant in space $A_{n}$ that is

$$
\varphi_{i, j}=0 .
$$

On the other hand, if in $A_{n}$ there exists a covariant constant vector field $\varphi_{i}$, then using of the substitution $\Psi(x)=-\ln (\Phi(x)), \partial_{i} \Phi(x)=\varphi(x)$ we can construct vector field $\psi_{i}(x)$, which satisfies (4.5) Thus Theorem 6 is proved.

In addition, we note that equation (4.9) forms linear system of Cauchy type in covariant derivatives with respect to unknown functions $\varphi_{i}(x)$. Integrability conditions of (4.9) has the form $\varphi_{\alpha} R_{i j k}^{\alpha}=0$. There are linear algebraic equations with respect to $\varphi_{i}(x)$. Their differential prolongations are also linear.

The global existence of these fields was discussed in [13].

\section{Preservation of Riemann AND RicCi TENSORS With RESPECT to THE SPECIAL CASE OF CANONICAL ALMOST GEODESIC MAPPINGS OF THE FIRST TYPE OF AFFINE CONNECTED SPACE}

Consider the special case of canonical almost geodesic mappings, which is characterized by the following equation

$$
P_{i j, k}^{h}+P_{i j}^{\alpha} P_{\alpha k}^{h}=\delta_{(i}^{h} a_{j k)},
$$

where $a_{i j}$ is some symmetric tensor.

Indeed, mappings which are characterized by equation (5.1), are canonical almost geodesic mappings of the first type, namely after symmetrization of equations (5.1) we obtain (2.5). 
For mappings, which are characterized by equations (5.1), tensor $A$ has the following structure

$$
A_{i j k}^{h}=\delta_{(i}^{h} a_{j k)},
$$

Then, obviously, conditions (3.3) and (3.5) are fulfilled for the tensor $A$. Therefore, there is a

Theorem 7. Riemannian and Ricci tensors are invariant geometrical objects under canonical almost geodesic mappings of the first type, which are characterized by (5.1).

Because in a projective-euclidean space Riemannian tensor vanishes, and in a Ricci-flat space Ricci tensor vanishes, then by Theorem (3.2) we have

Theorem 8. If projective euclidean or Ricci-flat space admits canonical almost geodesic mapping of the first type, which is characterized by equations (5.1), into space $\bar{A}_{n}$, then $\bar{A}_{n}$ is projective-euclidean or Ricci-flat space respectively.

Considering (5.1) as a Cauchy-type system with respect to deformation tensor $P_{i j}^{h}$ we can determine their condition of integrability.

For this we covariantly differentiate (5.1) on $x^{m}$, then we alternate it by indices $k$ and $m$. Using of the Ricci identity we have

$$
\begin{aligned}
\delta_{i}^{h} a_{j[k, m]}+\delta_{j}^{h} a_{i[k, m]}+ & \delta_{[k|i j| m}^{h}= \\
& -P_{i j}^{\alpha} R_{\alpha k m}^{h}+P_{\alpha(j}^{h} R_{i) k m}^{\alpha}+a_{j[m} P_{k] i}^{h}+a_{j[m} P_{k] j}^{h} .
\end{aligned}
$$

Contracting condition of integrability (5.3) by indices $h$ and $m$, we get

$$
\begin{aligned}
& a_{j k, i}+a_{i k, j}-(n+1) a_{i j, k}= \\
& \quad-P_{i j}^{\alpha} R_{\alpha k m}+P_{\alpha j}^{\beta} R_{j k \beta}^{\alpha}+a_{j \alpha} P_{k i}^{\alpha}-a_{j k} P_{\alpha i}^{\alpha}+a_{i \alpha} P_{j k}^{\alpha}-a_{i k} P_{j \alpha}^{\alpha} .
\end{aligned}
$$

We alternate equations (5.4) by indices $j$ and $k$, then we obtain

$$
\begin{aligned}
a_{i j, k}= & a_{i k, j}+\frac{1}{(n+2)}\left(P_{i j}^{\alpha} R_{\alpha k}+P_{i k}^{\alpha} R_{\alpha j}-P_{\alpha j}^{\beta} R_{i k \beta}^{\alpha}+P_{\alpha k}^{\beta} R_{i j \beta}^{\alpha}\right. \\
& \left.-P_{\alpha i}^{\beta} R_{j k \beta}^{\alpha}+P_{\alpha i}^{\beta} R_{k j \beta}^{\alpha}-a_{j \alpha} P_{k i}^{\alpha}+a_{k \alpha} P_{i j}^{\alpha}+a_{i k} P_{j \alpha}^{\alpha}-a_{i j} P_{k \alpha}^{\alpha}\right) .
\end{aligned}
$$

Observing (5.5), we can write equations in the following form

$$
\begin{aligned}
a_{i k, j}= & \frac{1}{(n-1)(n+2)}\left(n\left(P_{i k}^{\alpha} R_{\alpha j}-P_{\alpha(k}^{\beta} R_{i) j \beta}^{\alpha}\right)+R_{\alpha(k} P_{i) j}^{\alpha} R_{i j \beta}^{\alpha}-P_{\alpha j}^{\beta} R_{(i k) \beta}^{\alpha}\right. \\
& \left.-P_{\alpha(i}^{\beta} R_{|j| k) \beta}^{\alpha}+(n+1)\left(a_{j(i} P_{k) \alpha}^{\alpha}-a_{\alpha(i} P_{k) j}^{\alpha}\right)+2\left(a_{i k} P_{j \alpha}^{\alpha}-a_{j \alpha} P_{i k}^{\alpha}\right)\right) .
\end{aligned}
$$


Obviously, equations (5.1) and (5.6) in a given space represent a Cauchy type system with respect to functions $P_{i j}^{h}(x)$ and $a_{i j}(x)$ which, of course, are satisfied even algebraic conditions.

$$
P_{i j}^{h}(x)=P_{j i}^{h}(x), \quad a_{i j}(x)=a_{j i}(x) .
$$

So we proved

Theorem 9. An affine connected space $A_{n}$ admits almost geodesic mapping, defined by equation (5.1) onto affine connected space $\bar{A}_{n}$ if and only if there exist solution of mixed equation system of Cauchy type (5.1), (5.6) and (5.7) with respect to function $P_{i j}^{h}(x)$ and $a_{i j}(x)$.

The general solution of Cauchy type mixed system depends on (no more than) $\frac{1}{2} n(n+1)^{2}$ real parameters.

\section{REFERENCES}

[1] V. Berezovski, N. Guseva, and J. Mikeš, "On special first-type almost geodesic mappings of affine connection spaces preserving a certain tensor," Mat. Zametki, vol. 98, no. 3, pp. 463-466, 2015, doi: $10.4213 / \mathrm{mzm} 10817$.

[2] V. Berezovski and J. Mikeš, "On canonical almost geodesic mappings of the first type of affinely connected spaces," Russ. Math., vol. 58, no. 2, pp. 1-5, 2014, doi: 10.3103/S1066369X14020017.

[3] V. Berezovski and J. Mikeš, "Almost geodesic mappings of spaces with affine connection," $J$. Math. Sci., New York, vol. 207, no. 3, pp. 389-409, 2015.

[4] V. Berezovski, J. Mikeš, and A. Vanžurová, "Fundamental PDE's of the canonical almost geodesic mappings of type $\tilde{\pi}_{1}$," Bull. Malays. Math. Sci. Soc. (2), vol. 37, no. 3, pp. 647-659, 2014.

[5] V. Berezovski, S. Bácsó, and J. Mikeš, "Almost geodesic mappings of affinely connected spaces that preserve the Riemannian curvature," Ann. Math. Inform., vol. 45, pp. 3-10, 2015.

[6] V. Berezovski and J. Mikeš, "On a classification of almost geodesic mappings of affine connection spaces," Acta Univ. Palacki. Olomuc., Fac. Rerum Nat., Math., vol. 35, pp. 21-24, 1996.

[7] V. Berezovski, J. Mikeš, and A. Vanžurová, "Almost geodesic mappings onto generalized Riccisymmetric manifolds," Acta Math. Acad. Paedagog. Nyházi. (N.S.), vol. 26, no. 2, pp. 221-230, 2010.

[8] V. Berezovskij and J. Mikeš, "On the classification of almost geodesic mappings of affineconnected spaces," Differential geometry and its applications, Proc. Conf., Dubrovnik/Yugosl. 1988, 41-48, 1989.

[9] V. Berezovsky and J. Mikeš, "On special almost geodesic mappings of type $\pi_{1}$ of spaces with affine connection," Acta Univ. Palacki. Olomuc., Fac. Rerum Nat., Math., vol. 43, pp. 21-26, 2004.

[10] I. Hinterleitner and J. Mikeš, "Geodesic mappings of (pseudo-) Riemannian manifolds preserve class of differentiability," Miskolc Math. Notes, vol. 14, no. 2, pp. 575-582, 2013.

[11] I. Hinterleitner and J. Mikeš, "Geodesic mappings and differentiability of metrics, affine and projective connections," Filomat, vol. 29, no. 6, pp. 1245-1249, 2015, doi: 10.2298/FIL1506245H.

[12] I. Hinterleitner and J. Mikeš, "Geodesic mappings and Einstein spaces," in Geometric methods in physics. XXX workshop. Basel: Birkhäuser, 2013, pp. 331-335.

[13] J. Mikeš and M. Chodorová, "On concircular and torse-forming vector fields on compact manifolds,” Acta Math. Acad. Paedagog. Nyházi. (N.S.), vol. 26, no. 2, pp. 329-335, 2010. 
[14] J. Mikeš and H. Chudá, "On geodesic mappings with certain initial conditions," Acta Math. Acad. Paedagog. Nyházi. (N.S.), vol. 26, no. 2, pp. 337-341, 2010.

[15] J. Mikeš, A. Vanžurová, and I. Hinterleitner, Geodesic mappings and some generalizations. Olomouc: Palacký University, Faculty of Science, 2009.

[16] J. Mikeš, "Geodesic mappings of affine-connected and Riemannian spaces," J. Math. Sci., New York, vol. 78, no. 3, pp. 311-333, 1996.

[17] J. Mikeš, "Holomorphically projective mappings and their generalizations," J. Math. Sci., New York, vol. 89, no. 3, pp. 1334-1353, 1998.

[18] J. Mikeš and V. Berezovski, "Geodesic mappings of affine-connected spaces onto Riemannian spaces," in Differential geometry and its applications. Proceedings of a colloquium, held in Eger, Hungary, August 20-25, 1989, organized by the János Bolyai Mathematical Society. Amsterdam: North-Holland Publishing Company; Budapest: János Bolyai Mathematical Society, 1992, pp. 491-494.

[19] Mikeš J. at al, Differential geometry of special mappings. Olomouc: Palacký University, Faculty of Science, 2015.

[20] N. Sinyukov, "Geodesic mappings of Riemannian spaces," 1979.

[21] M. S. Stanković, M. L. Zlatanović, and N. O. Vesić, "Basic equations of $G$-almost geodesic mappings of the second type, which have the property of reciprocity," Czech. Math. J., vol. 65, no. 3, pp. 787-799, 2015.

[22] E. S. Stepanova, J. Mikeš, and I. I. Tsyganok, "A geodesic mapping and its field of symmetric linear endomorphisms," Diff. Geom. Appl., vol. 35, pp. 44-49, 2014, doi: 10.1016/j.difgeo.2014.03.006.

[23] K. Yano, "Differential geometry on complex and almost complex spaces," Oxford-London-New York-Paris-Frankfurt: Pergamon Press. XII, 323 p. (1965)., 1965.

[24] M. L. Zlatanović, L. S. Velimirović, and M. S. Stanković, "Necessary and sufficient conditions for equitorsion geodesic mapping," J. Math. Anal. Appl., vol. 435, no. 1, pp. 578-592, 2016, doi: 10.1016/j.jmaa.2015.10.052.

\section{Authors' addresses}

\section{E. Berezovski}

Uman National University of Horticulture, Dept. of Mathematics, Instytutska 1, Uman, Ukraine,

E-mail address: berez.voloderambler.ru

\section{S. Bácsó}

University of Debrecen, 4010 Debrecen, Hungary,

E-mail address: bacsos@unideb.hu

\section{J. Mikeš}

Palacky University, Dept. of Algebra and Geometry, 17. listopadu 12, 77146 Olomouc, Czechia, E-mail address: josef.mikes@upol.cz 